\title{
Fighting infant infections with myeloid-derived suppressor cells
}

\author{
Rebekka Weber and Viktor Umansky \\ Skin Cancer Unit, German Cancer Research Center (DKFZ), Heidelberg and Department of Dermatology, Venereology and Allergology, University Medical Center Mannheim, Ruprecht Karl University of \\ Heidelberg, Mannheim, Germany
}

\begin{abstract}
Recent work demonstrated a role for myeloid-derived suppressor cells (MDSCs) in the antimicrobial response in newborns, but the signals guiding their differentiation remained unknown. In this issue of the JCI, Liu et al. demonstrate that lactoferrin (LF) converts newborn neutrophils and monocytes to MDSCs via the low-density lipoprotein receptor-related protein-2 (LRP2) receptor and NF-KB activation. Due to their strong antimicrobial activity, adoptive transfer of MDSCs generated by in vitro culture with LF prolonged the survival of newborn mice with necrotizing enterocolitis, a severe pathology in preterm infants. These findings indicate a surprising protective role of MDSCs in newborns and demonstrate the potential of MDSC therapy for the treatment of infants with diseases associated with deregulated inflammation.
\end{abstract}

\section{MDSCs can be generated under} physiological conditions

Myeloid-derived suppressor cells (MDSCs) represent a heterogeneous population of immature myeloid cells with broadly distinct phenotypes that fail to terminally differentiate into granulocytes, macrophages, or DCs. They exhibit a remarkable capacity to inhibit immune responses mediated by T, B, and NK cells by different mechanisms, including increased production of reactive oxygen species and nitric oxide (NO) as well as upregulated expression of arginase-1, prostaglandin E2 (PGE2), and programmed cell death ligand-1 (1, 2). MDSCs comprise two major subsets: polymorphonuclear (PMN-MDSCs) and monocytic (M-MDSCs), which share characteristics with neutrophils and monocytes, respectively $(1,2)$. MDSCs are generated in chronic inflammatory conditions characterized by long-term secretion and accumulation of soluble inflammatory factors, including IL-1 $\beta$, IL-6, TNF- $\alpha$,
$\operatorname{IFN}-\gamma$, and GM-CSF $(2,3)$. By recruiting and activating MDSCs, these factors create a feedback loop that restricts excessive immune reactions. However, in pathological situations, such as chronic infection or cancer, MDSCs induce a profound immunosuppression and may therefore support tumor progression. In addition to soluble inflammatory mediators, MDSCs can also be generated from immature or mature myeloid cells in mice and humans upon exposure to extracellular vesicles secreted by tumor cells $(4,5)$.

It was long believed that MDSCs are only present in the context of pathological disorders and pregnancy and are absent under steady-state conditions in healthy hosts (2). However, recent observations demonstrated MDSC accumulation in healthy neonates $(6,7)$. Interestingly, MDSCs from newborns were found to inhibit $\mathrm{T}$ cell activity in a way similar to those from cancer or chronically infected patients $(6,7)$, but in contrast to cancer

\section{Related Article: p. 4261}

Conflict of interest: The authors have declared that no conflict of interest exists.

MDSCs, neonatal mouse MDSCs display a strong potential to kill pathogens (7). Moreover, PMN-MDSCs obtained from term neonates exhibit strong phagocytic activity as well as decreased apoptosis and immunosuppressive properties after bacterial infection (8). These data suggest a critical role of MDSCs in preventing newborns from inflammatory diseases.

The first weeks after birth are characterized by high vulnerability to diseases associated with dysregulated inflammation, such as bronchopulmonary dysplasia, necrotizing enterocolitis (NEC), and sepsis. NEC is the most common gastrointestinal emergency in preterm infants, with a high mortality rate (9). The disorder is characterized by ischemic necrosis of the intestinal mucosa, severe bowel and systemic inflammation, and dissection of gas into the bowel wall and portal venous system (9). The systemic inflammatory response associated with the acute intestinal injury in NEC leads to the impairment of multiple organs, with a high risk for chronic deficits in lung and renal function (10). Although it was previously demonstrated that MDSCs could be an important component of natural host defense mechanisms in newborns (6-8), the clinical significance of these cells and the molecular mechanisms of their generation in preterm infants remained unclarified.

\section{How MDSCs accumulate in healthy neonates}

In this issue of the JCI, a study by Liu and colleagues provides new insights into the molecular mechanisms of MDSC accumulation in newborns and brings a different perspective on the role of these cells in the protection of newborns from pathological inflammatory processes (11). The study addresses the paradoxical finding that such a powerful immunosuppressive cell population could exert a strong antibacterial activity. To resolve this paradox, the authors hypothesized that MDSCs 


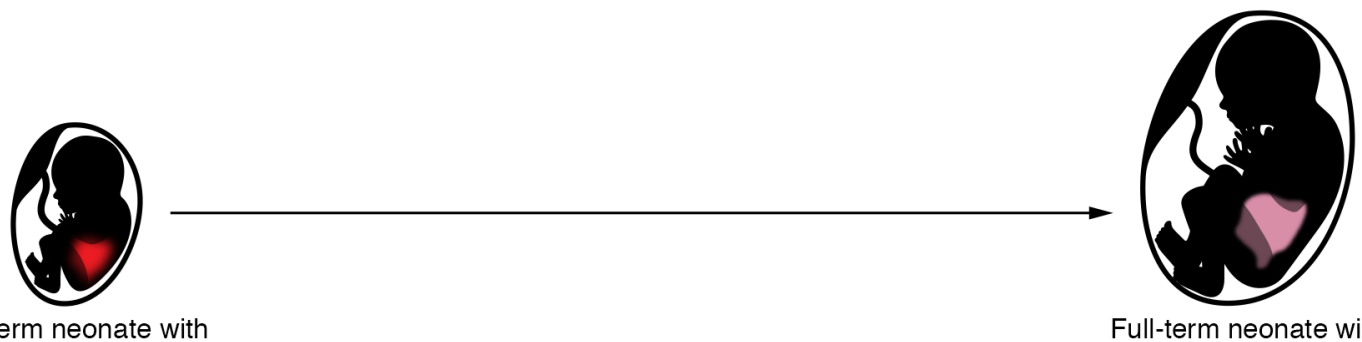

Preterm neonate with

Full-term neonate with inflamed intestine

healthy intestine
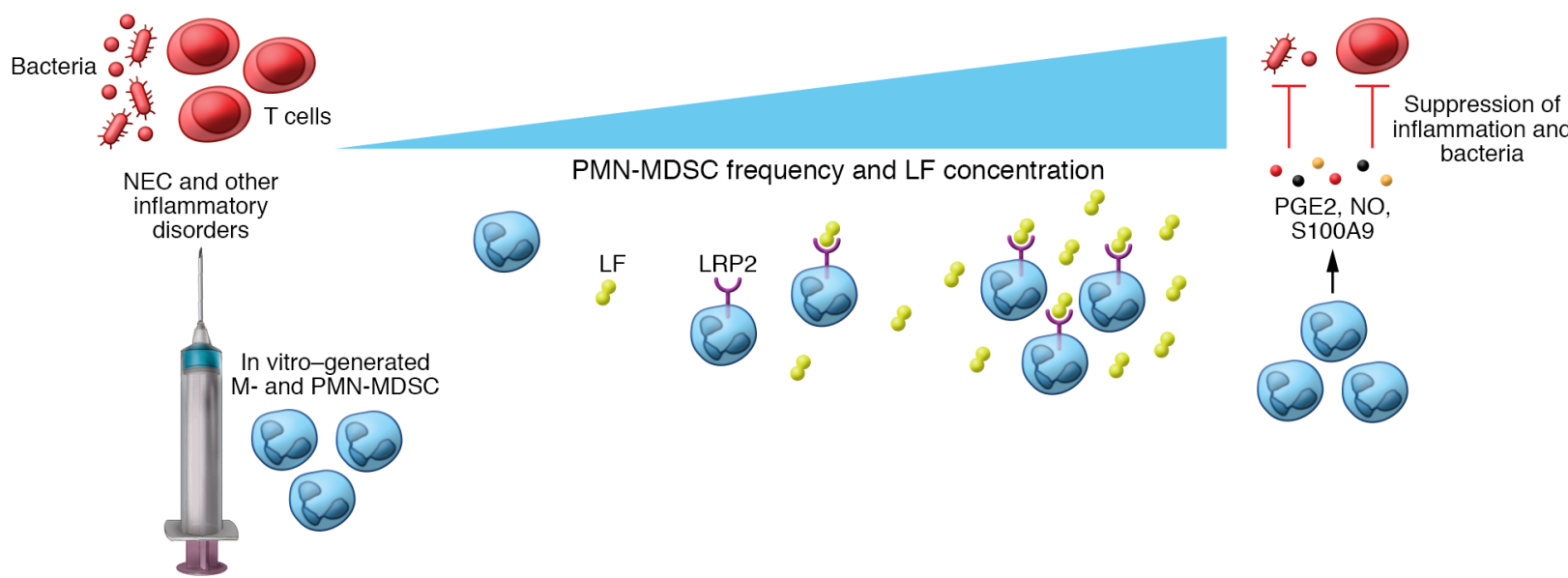

Figure 1. MDSCs protect newborns from inflammatory disorders. Preterm neonates show a lower abundance of PMN-MDSCs and LF as compared with full-term neonates. The amount of PMN-MDSCs and LF is further decreased in preterm neonates with NEC as compared with those without this disease. PMN-MDSCs in healthy newborns produce NO, PGE2, and S100A9, suppressing T cell activities and exerting simultaneously antibacterial properties. MDSCs could be generated in vitro from newborn (but not adult) monocytes and neutrophils via the binding of LF to its receptor LRP2 on these cells. Mice with different pathological inflammatory conditions were successfully treated with in vitro-generated MDSCs, suggesting that such cell therapy could be efficient in newborns with NEC and other inflammatory disorders.

devolved to control the severe inflammation associated with seeding of the gut microbiota during the first week of life. They first demonstrated that the frequency of PMN-MDSCs in the blood of preterm infants was significantly lower than that observed in full-term infants. Moreover, the decreased frequency of MDSCs in preterm infants was associated with development of NEC (11).

This group previously reported that lactoferrin (LF) in mother's milk plays a critical role in the accumulation of MDSCs in newborns (7). LF is a member of the transferrin family, a group of iron-binding proteins found in many eukaryotic organisms (12). Studies in knockout mice have shown that LF is not absolutely required as an iron source for infants $(13,14)$. However, LF biosynthesis can be stimulated during certain bacterial infections (14), and it seems to exert both immunostimulatory and immunoregulatory activities $(13,14)$. For instance, LF can bind bacterially released endotoxin to prevent overstimulation of the immune system (14), but has also been shown to stimulate maturation of dendritic cells and to recruit various subsets of leukocytes $(14,15)$.

In line with their previous findings that LF can promote accumulation of MDSCs in newborns (7), in the current study, Liu et al. report that the amount of LF in serum is strongly correlated with the frequency of PMN-MDSCs in neonates (11). Moreover, they demonstrate that neutrophils and monocytes from mouse and human (both full term and preterm) neonates can be converted by LF to potent immunosuppressive MDSCs through stimulation of NF- $\kappa$ B. Importantly, Liu et al. show that LF strongly activates the antibacterial properties of MDSCs. To determine the molecular mechanism of the LF effects, the authors measured the expression of the primary LF receptor, low-density lipoprotein receptor-related protein-2 (LRP2) (16), and found that its expression gradually decreased with age in mouse and human newborns. Although LRP2 expression in preterm infants was lower than in full-term neonates, it was still substantially higher than in adults, allowing the generation of MDSCs from preterm neonate myeloid cells upon exposure to LF. Interestingly, upregulating LRP2 rendered adult myeloid cells sensitive to MDSC conversion by LF treatment (11).

\section{Therapeutic implications and conclusions}

The authors next tested the therapeutic potential of these cells in a mouse model of NEC. They found that adoptive transfer of LF-induced MDSCs significantly decreased bacterial load in recipient mice, prolonging their survival (11). Moreover, LF-MDSCs demonstrated potent antiinflammatory properties in other experimental models of inflammation observed in adults, such as autoimmune lung inflammation, colitis, and hepatitis. Since adult myeloid cells can be manipulated in vitro to render them sensitive to LF treatment, it may be possible to utilize LF-MDSCs therapeutically, not only in neonates, but also in adults. Intriguingly, the present study also shows that treatment with LF-induced MDSCs is more effective 
in the mouse model of NEC than treatment with LF alone (11).

Taken together, the experimental findings described in this timely and innovative manuscript provide important insights into our knowledge of the role of MDSCs in healthy neonates. Whereas the detrimental function of MDSCs in cancer was clearly established by their capacity to inhibit antitumor reactivity of $\mathrm{T}$ and $\mathrm{NK}$ cells, MDSCs in newborns seem to play a positive role. After conversion from neonate neutrophils and monocytes by LF via its receptor LRP2, MDSCs acquired not only strong immunosuppressive activity, but also potent antiinflammatory and antibacterial properties, protecting newborns from severe morbidities (Figure 1). Importantly, adult myeloid cells can be manipulated to respond to LF and acquire neonatal MDSC properties and could be potentially applied to treat pathological conditions in adults, such as autoimmune lung inflammation, colitis, and hepatitis. Future studies should clarify whether the immunosuppressive function of MDSCs interferes with their antibacterial and antiinflammatory properties in adults.

\section{Acknowledgments}

This Commentary was partially supported by grants from the German Research
Council (RTG2099 to RW and VU) and the DKFZ-MOST Cooperation in Cancer Research (CA181 to VU).

Address correspondence to: Viktor Umansky, Skin Cancer Unit (A370), German Cancer Research Center, Im Neuenheimer Feld 280, 69120 Heidelberg, Germany. Phone: 49.621.3833773; Email: v.umansky @dkfz-heidelberg.de.

1. Bronte V, et al. Recommendations for myeloidderived suppressor cell nomenclature and characterization standards. Nat Commun. 2016;7:12150.

2. Veglia F, Perego M, Gabrilovich D. Myeloidderived suppressor cells coming of age. Nat Immunol. 2018;19(2):108-119.

3. Ben-Meir K, Twaik N, Baniyash M. Plasticity and biological diversity of myeloid derived suppressor cells. Curr Opin Immunol. 2018;51:154-161.

4. Huber V, et al. Tumor-derived microRNAs induce myeloid suppressor cells and predict immunotherapy resistance in melanoma. J Clin Invest. 2018;128(12):5505-5516.

5. Fleming V, et al. Melanoma extracellular vesicles generate immunosuppressive myeloid cells by PD-L1 upregulation via TLR4 signaling. Cancer Res. doi:10.1158/0008-5472.CAN-19-0053.

6. Schwarz J, et al. Granulocytic myeloid-derived suppressor cells (GR-MDSC) accumulate in cord blood of preterm infants and remain elevated during the neonatal period. Clin Exp Immunol. 2018;191(3):328-337.

7. He YM, et al. Transitory presence of myeloidderived suppressor cells in neonates is crit- ical for control of inflammation. Nat Med. 2018;24(2):224-231.

8. Leiber A, et al. Neonatal myeloid derived suppressor cells show reduced apoptosis and immunosuppressive activity upon infection with Escherichia coli. Eur J Immunol. 2017;47(6):1009-1021.

9. Niño DF, Sodhi CP, Hackam DJ. Necrotizing enterocolitis: new insights into pathogenesis and mechanisms. Nat Rev Gastroenterol Hepatol. 2016;13(10):590-600.

10. Hintz SR, et al. Neurodevelopmental and growth outcomes of extremely low birth weight infants after necrotizing enterocolitis. Pediatrics. 2005;115(3):696-703.

11. Liu Y, et al. Lactoferrin-induced myeloid-derived suppressor cell therapy attenuates pathologic inflammatory conditions in newborn mice. JClin Invest. 2019;129(10):4261-4275.

12. Lambert LA, Perri H, Halbrooks PJ, Mason AB. Evolution of the transferrin family: conservation of residues associated with iron and anion binding. Comp Biochem Physiol B, Biochem Mol Biol. 2005;142(2):129-141.

13. Ward PP, Paz E, Conneely OM. Multifunctional roles of lactoferrin: a critical overview. Cell Mol Life Sci. 2005;62(22):2540-2548.

14. Vogel HJ. Lactoferrin, a bird's eye view. Biochem Cell Biol. 2012;90(3):233-244.

15. de la Rosa G, Yang D, Tewary P, Varadhachary A, Oppenheim JJ. Lactoferrin acts as an alarmin to promote the recruitment and activation of APCs and antigen-specific immune responses. J Immunol. 2008;180(10):6868-6876.

16. Grey A, et al. The low-density lipoprotein receptorrelated protein 1 is a mitogenic receptor for lactoferrin in osteoblastic cells. MolEndocrinol. 2004;18(9):2268-2278. 